\title{
Vol. 70, No. 4
}

In the report "Trends in Outbreak-Associated Cases of COVID-19 - Wisconsin, March-November 2020," on page 114, the third sentence of the second paragraph should have read "Confirmed cases of COVID-19 that were linked" to these outbreaks were analyzed by symptom onset date (or sample collection date) and the reported setting of the associated outbreaks during three periods: before and during Wisconsin's Safer At Home order** (March 4-May 12), summer and return-to-school (May 13-September 2), and the exponential growth phase ${ }^{\dagger \dagger}$ (September 3-November 16)." 